\title{
Usefulness of Buddy Wire Technique During Transcatheter Transseptal Mitral Valve Implantation in Degenerated Mitral Bioprosthesis
}

Daisuke Hachinohe, MD; Azeem Latib, MD; Satoru Mitomo, MD;

Matteo Montorfano, MD; Antonio Colombo, MD

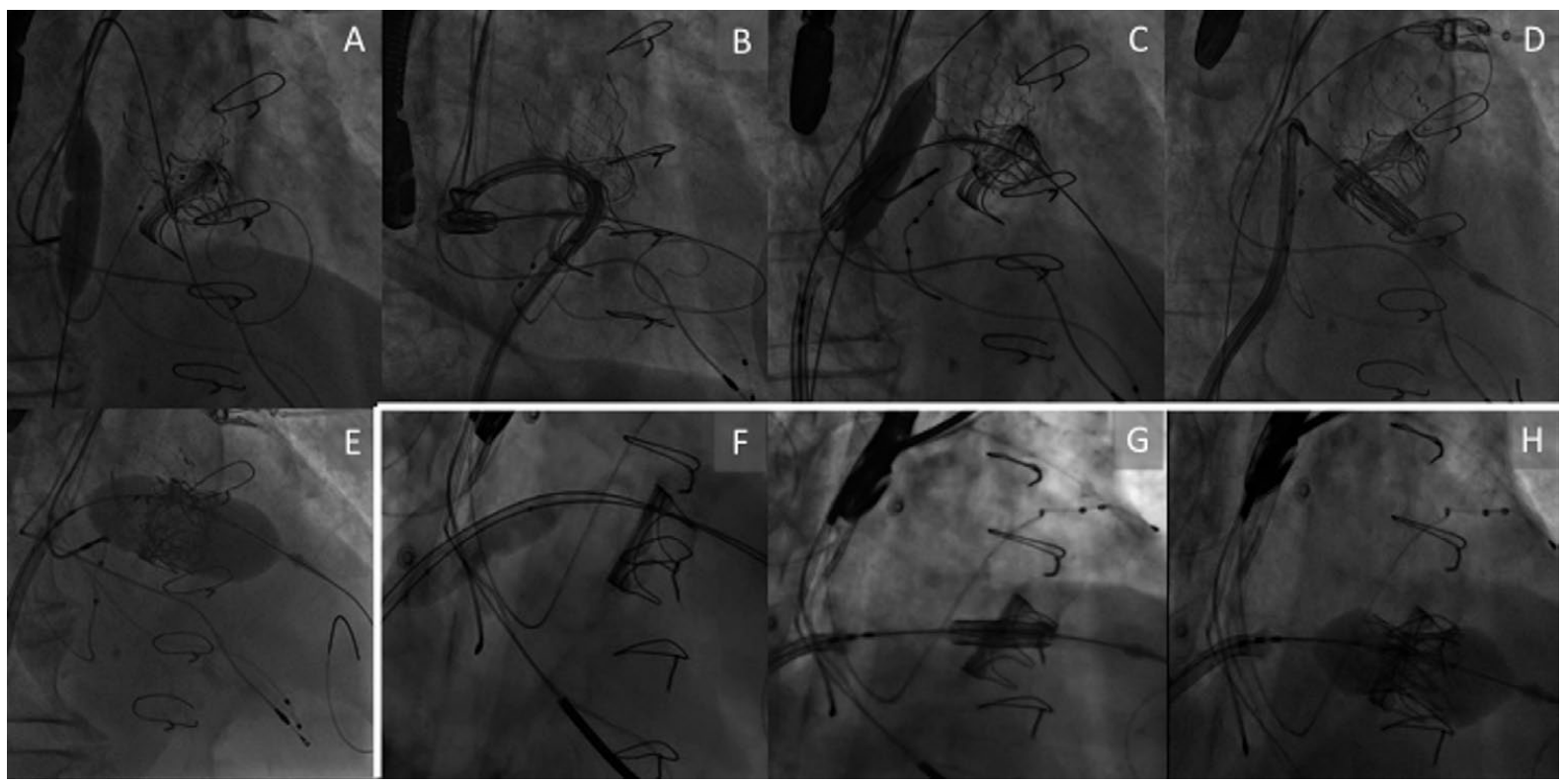

Figure. Transcatheter mitral valve (29-mm SAPIEN3) implantation in degenerated surgical mitral prosthesis (A-E, 29-mm Perimount; case 1; F-H, 27-mm Perimount; case 2). (A) A 14×40-mm peripheral balloon was dilated at the interatrial septum (IAS) with one Safari small wire (SSW). (B) A 29-mm SAPIEN3 transcatheter heart valve (THV) could not cross the IAS in spite of changing directions of a guiding system (Movie S1). (C) After another transseptal puncture, the IAS was re-dilated through an SSW placed in the left atrium (LA). (D) The THV could be crossed into the LA and the surgical prosthesis with considerable resistance (Movie S1). (E) The THV was implanted during rapid pacing. (F) Dilatation of the IAS with two SSW placed in the LV. (G) The THV could easily cross the IAS and the surgical bioprosthesis (Movie S2). (H) The THV was implanted.

A n 82-year-old man had heart failure due to severe stenosis of surgical aortic and mitral bioprosthesis. After transcatheter aortic valve implantation (TAVI) in the failed surgical aortic bioprosthesis, the patient underwent transcatheter mitral valve implantation (TMVI) in the failed surgical mitral bioprosthesis (Edwards Perimount $29 \mathrm{~mm}$, Edwards Lifesciences, USA) via the transseptal approach. One Safari small wire (SSW, Boston
Scientific, USA) was placed into the left ventricle (LV). A $14 \times 40 \mathrm{~mm}$ peripheral balloon dilated the interatrial septum (IAS; Figure A). A 29-mm SAPIEN3 (Edwards Lifesciences) transcatheter heart valve (THV) failed to cross despite several attempts to get stronger support of the guiding system (Figure B; Movie S1). Replacement of SSW with a Lunderquist Extra-Stiff Wire Guide (Cook, USA) did not resolve the problem. After another transseptal puncture,

Received April 16, 2018; revised manuscript received May 7, 2018; accepted May 23, 2018; J-STAGE Advance Publication released online June 23, 2018 Time for primary review: 20 days

Interventional Cardiology Unit, San Raffaele Scientific Institute, Milan (D.H., A.L., S.M., M.M., A.C.); Interventional Cardiology Unit, EMO-GVM Centro Cuore Columbus, Milan (D.H., A.L., S.M., A.C.), Italy

Mailing address: Antonio Colombo, MD, IRCCS San Raffaele Scientific Institute, Via Olgettina 60, 20132 Milan, Italy. E-mail: colombo.antonio@hsr.it

ISSN-1346-9843 All rights are reserved to the Japanese Circulation Society. For permissions, please e-mail: cj@j-circ.or.jp 
an SSW was crossed into the left atrium (LA). After re-dilatation of the IAS through the SSW (Figure C), the THV could be passed into the LA and the bioprosthesis with some resistance (Figure D; Movie S1), and implanted (Figure E).

In a subsequent case, a 75-year-old woman underwent TMVI in an Edwards Perimount 27-mm. Two SSW were initially placed in the LV. IAS was dilated with a $14 \times 40$ $\mathrm{mm}$ balloon (Figure F), and a $29-\mathrm{mm}$ SAPIEN 3 could be easily crossed through the IAS and the surgical bioprosthesis (Figure G; Movie S2), and deployed after removing oner wire (Figure H).

A buddy wire technique can offer better support to facilitate balloon dilatation of the IAS, passage of the THV across the septum, re-dilatation of the septum if the THV cannot cross, and facilitate a buddy balloon technique if the THV cannot cross the degenerated surgical prosthesis.

\section{Disclosures}

A.L. has served on the advisory board for Medtronic; and on the
Speakers Bureau for Abbott Vascular. M.M. is a proctor for Edwards Lifesciences and Abbott Vascular. The other authors declare no conflicts of interest.

\section{Acknowledgments / Sources of Funding}

None.

\section{Supplementary Files}

\section{Supplementary File 1}

Movie S1. A 29-mm SAPIEN3 transcatheter heart valve (THV) could not cross to interatrial septum (IAS) in spite of changing directions of a guiding system. Finally, the THV could be crossed into left atrium and the surgical prosthesis with considerable resistance.

\section{Supplementary File 2}

Movie S2. The THV could be easily crossed through the IAS and the surgical bioprosthesis.

Please find supplementary file(s); http://dx.doi.org/10.1253/circj.CJ-18-0413 